\title{
Automatic Leukocyte Classification Based on Microscope Images
}

\author{
Piyamas Suapang a,", Methinee Thongyoun ${ }^{\mathrm{b}}$, Sorawat Chivapreecha ${ }^{\mathrm{c}}$ \\ ${ }^{a}$ Biomedical Engineering Program, Department of Physics, Rangsit University, Pathumthani, 12000, Thailand \\ ${ }^{b, c}$ Department of Telecommunications Engineering, King Mongkut's Institute of Technology Ladkrabang, Bangkok, \\ 10520, Thailand \\ * piyamas_suapang@yahoo.com
}

\begin{abstract}
Numbers of white blood cells in different classes help doctors to diagnose patients. A technique for automating the differential count of white blood cell is presented. The proposed system takes an input, color image of stained peripheral blood smears. The process involves segmentation, feature extraction and classification. The segmentation procedure, a novel simple algorithm, is proposed for localization of white blood cells and the different cell components are separated with automatic thresholding. Features extracted from the segmented nucleus are motivated by the visual cues of shape, color and texture. This research uses the artificial neural network for implemention and uses the different combinations of feature sets. The results presented here are based on trials conducted with normal cells. For training the classifiers, a library set of 233 patterns is used. The tested data consists of 134 samples and produced correct classification rate close to $88.10 \%$.
\end{abstract}

Keywords: leukocyte classification, segmentation, artificial neural network.

\section{Introduction}

A typical blood microscope image has been digitalized by a CCD and acquired by a frame-grabber system [1]. The microscope inspection of blood slides provides important qualitative and quantitative information concerning the presence of hematic pathologies as shown in figure 1 . Principal cells present in the blood are red blood cells, and the white cells (leucocytes). Leucocyte cells containing granules are called granulocytes (composed by neutrophil, basophil, eosinophil). Cells without granules are called agranulocytes (lymphocyte and monocyte). The percentage of leucocytes in human blood typically ranges between the following values: neutrophils $50-70 \%$. eosinophils $1-5 \%$. basophils $0-1 \%$. monocytes $2-10 \%$, lymphocytes $20.45 \%$ [2]. These cells provide the major defense against infections in the organism and their specific concentrations can help specialists to discriminate the presence or not of very important families of pathologies (i.e. the presence of mononucleosis, hepatitis diabetes, allergy, arthritis, anaemia, and many others).

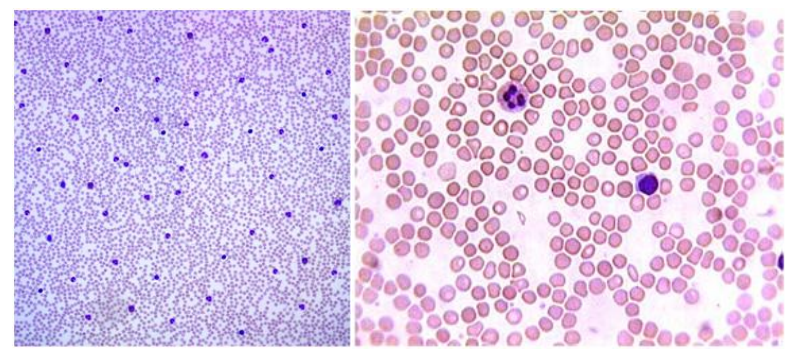

Fig. 1. A typical blood microscope image.

Some typical examples of these types are shown in figure 2, where neutrophils has small granules in cytoplasm and only one nucleus, with a variable number of lobes; eosinophils has bilobed nucleus and coarse cytoplasmic granules; basophils include many cytoplasmic granules over the nucleus; monocytes has a kidney-shaped nucleus and slightly basophilic in the cytoplasm and lymphocytes has round nucleus and is devoid of specific granules. Table I summarizes the characteristic features of these cells and their relative size and number in normal blood.

From decades this operation is performed by experienced operators, which basically perform two main analyses. The first is the qualitative study of the morphology of the cells and it gives information of 
degenerative and tumoral pathologies such as leukemia. The second approach is quantitative and it consists of differential counting the white blood's cells. Unfortunately, the accuracy of cell classification and counting is strongly affected by individual operator's capabilities. In particular, the identification and the differential count of blood's cells is a time-consuming and repetitive task that can be influenced by the operator's accuracy and tiredness. The automated classification of the peripheral white blood cells (leukocytes) has been the subject of this study. The peripheral blood leukocytes provide a very interesting and challenging medium for the study for biological image processing and classification. The taxonomy of the various blood cells has had a classic history in histology [3], and a qualitative, verbally descriptive taxonomy has been established in the hematological literature [4]. One of the rapidly emerging areas of pattern recognition has been medical picture processing and image classification [5], [6]. Radioisotope scanning [7], [8], breast cancer diagnosis [9], the classification of Papanicolaou smears [10], chromosome analysis [11], [12], and the classification of white blood cells [13-16], have all proven to some extent to be amenable to pattern recognition techniques.

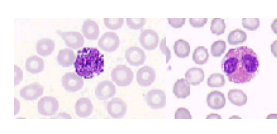

(a) (b)

(c)

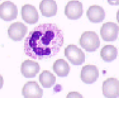

(e)
Fig. 2 Examples of five major groups of human leukocytes in peripheral blood: (a) Basophil (b) Eosinophil (c) Lymphocyte (d) Monocyte and (e) Neutrophil.

Table 1. The features of Leukocytes.

\begin{tabular}{|l|l|l|l|l|l|}
\hline \multirow{2}{*}{ Features } & \multicolumn{3}{|c|}{ Granulocytes } & \multicolumn{2}{c|}{ Agranulocytes } \\
\cline { 3 - 6 } & Neutrophil & Eosinophil & Basophil & Monocyte & Lymphocyte \\
\hline Diameter & $12-15 \mu \mathrm{m}$ & $12-15 \mu \mathrm{m}$ & $12-15 \mu \mathrm{m}$ & $12-20 \mu \mathrm{m}$ & $6-18 \mu \mathrm{m}$ \\
\hline & U-shaped, & Segmented, & Poorly & Kidney & Round \\
\hline Nucleus & S-shaped, & bilobed & shown, & shaped & \\
\hline & 2-5 & & S-shaped & & \\
\hline & segmented & & & & \\
\hline & Azurophilic & Eosinophilic & Basophilc & Basophilic & Scanty, light \\
\hline & granules; & granules & granules & bluish-gray & blue \\
\hline Granules & Specific & & of & & \\
& granules & & different & & \\
\hline & & & sizes & & \\
\hline
\end{tabular}

The goal of this research is to determine whether all steps in a fully automated system for the classification of white blood cells from microscopic images can be realized using image processing and supervised learning techniques. As a component of this, we will attempt to determine which of a number of investigated classification techniques provides the best automated classifier for the classification of white blood cells into their five major types (Neutrophils, Lymphocytes, Monocytes, Eosinophils and Basophils), based on a limited data set of visual images. This paper focuses on the automated detection and classification (the haematologists' term for this process is differentiation) of white blood cells from color images captured from a microscope. Figure 3 shows useful regions of interest, each centered on a white blood cell, can be automatically extracted from color microscopic images. Secondly, image processing techniques can be used to automatically extract a feature set from these regions of interest that is useful for the classification of white blood cells. Finally, supervised classification techniques such as neural networks can be used to classify the white blood cells in these regions of interest and their relative accuracies can be compared.

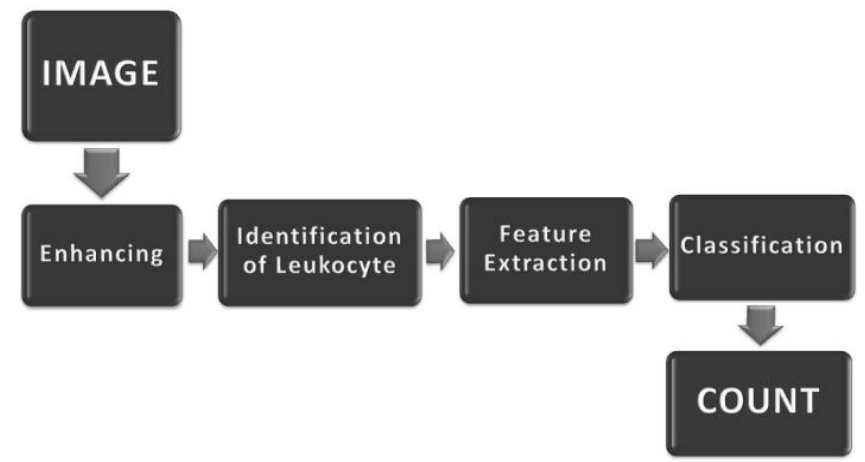

Fig. 3. The structure of modules composing the leukocytes classification system.

\section{Methodology}

\subsection{Segmentation}

First, the captured image file was split into its three component bands (red green and blue as shown in figure 4). The result was three grayscale files one for each of the red green and blue components of the image captured by the camera. Histogram analysis was used to examine three grayscale components (corresponding to the red, green and blue bands) of 30 images covering all five basic white blood cell types. It was found that the green component was consistently a better discriminator between the purple nuclear material and the rest of the image.

We did utilize thresholding to produce a binary bitmap 
image from the green band bitmap for each image. An automatic threshold value was selected by minimum value to discriminate between nuclear and nonnuclear pixels, as every white blood cell has a nucleus. We used only the green band for this nuclear thresholding, as, in this band, the nuclear material is much darker than either the cytoplasm (with the exception of basophilic cytoplasm) or the background. Experimentation showed that an automatic threshold value of 95 (on a scale of 0-256) gave an acceptable discrimination between nuclear and non-nuclear pixels, leaving nuclear pixels black on a white background as shown in figure 5 .

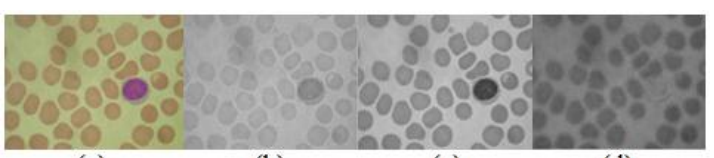

(a)

(b)

(c)

(d)

Fig. 4. (a) Raw captured image and (b, c, d) 3 single color components.
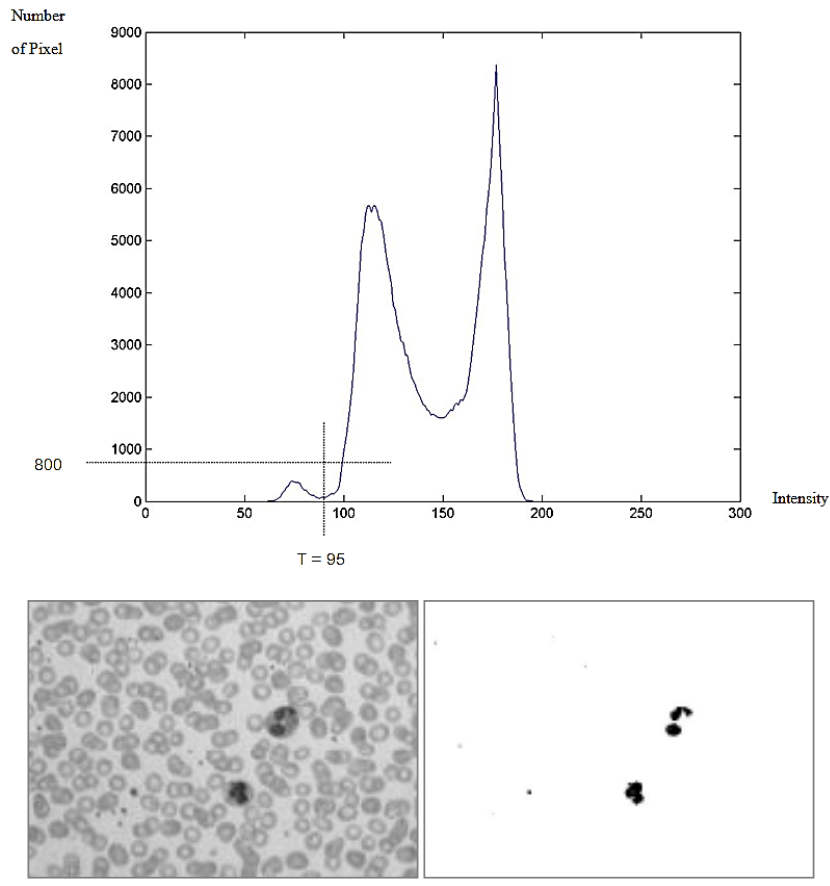

Fig. 5. Green component image and thresholded bitmap.

Due to edge effects on the captured images (the camera produced noticeable dark bands at the very edges of the images, as seen in Figure 4 and Figure 5), there were many nonnuclear pixels around the perimeter of the image. An edge erosion filter, which simply set all of the pixels within 3 pixels of the edge of the image to white, was developed and used. This removed the dark bands around the edge of the image as shown in figure 6 .

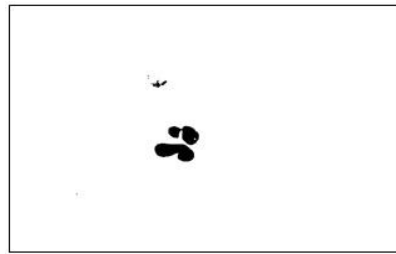

(a)

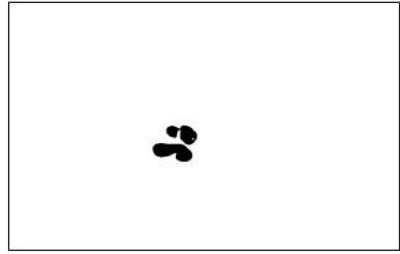

(b)
Fig. 6. The results of (a) thresholding and (b) erosion.

As a first step towards identifying a region of interest, an algorithm was developed to identify blobs (continuous connected groups of black - presumably nuclear - pixels) within a bitmap file and print out their details, including number of pixels in the blob and centroid (arithmetic mean of the $\mathrm{x}$ and $\mathrm{y}$ position values) of the blob. The $\mathrm{x}$ value of the centroid was found by summing the $\mathrm{x}$ values of all pixels in a blob and dividing the sum by the number of points in the blob. The y value of the centroid was found in a similar fashion, summing the $y$ values of all pixels in the blob and dividing by the number of pixels. The (x, y) location represented by this centroid was used as the centre of the blob of nuclear material. The centre (cx, cy) and number of points were recorded for each blob found.

A Region of interest (ROI) was defined around the centre (cx, cy) of each blob. This region of interest was defined to be a rectangle 110 by 110 pixels in size. The size of the ROI was originally chosen to preserve the aspect ratio of the camera's image, be large enough to accommodate the largest leukocyte with some headroom. The rectangular aspect ratio was later found to be unnecessary, a square ROI would have been simpler. This process produced a series of smaller images like the one in figure 7 .

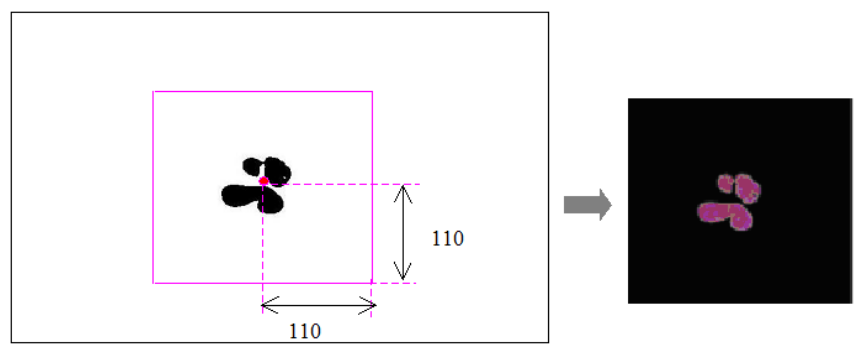

Fig. 7. Extracted image of white blood cell.

Most white blood cells are roughly circular in shape, though some (monocytes, in particular) may deviate significantly from the circular). For the purposes of obtaining a good feature set, representative of the features of the cell only, we chose to try to find the largest circular 
area entirely within the cell. This process produced finding circle as shown in figure 8 .

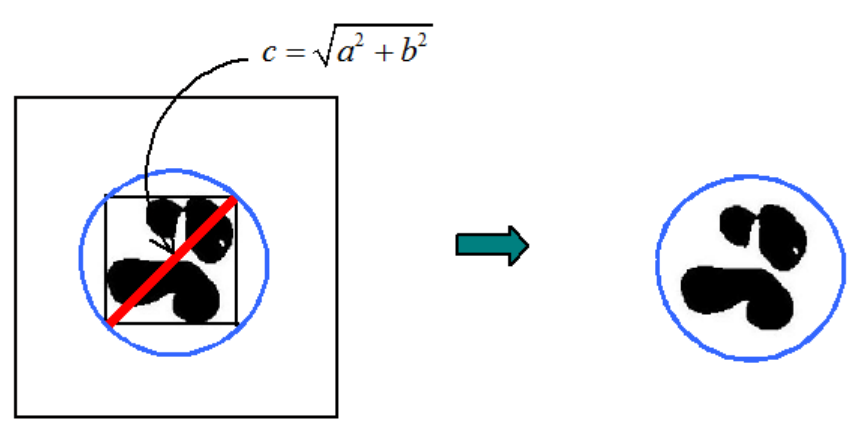

Fig. 8. Finding circle.

\subsection{Feature Extraction}

The segmented circular cell region was processed on a pixel by pixel basis and statistical information (mean, standard deviation, maximum value and minimum value) was collected for each of 5 color bands. The color bands were the red, green and blue captured by the hardware (camera + composite video capture card) together with the color ratios green/red and green/blue. The number of pixels within the circle was also determined. The red, blue and green band pixel values were just the 8 bit (0-255) values captured by Matrox Morphis (MOR/2VD/84*) capture card. The green/blue color ratio for a given pixel was determined by dividing the green band value by the blue band value. The green/red value was similarly obtained by dividing the red band value by the blue band value.

\subsection{Neural Network Architecture for Pattern Classification}

The neural networks are processing structures "consisting of many interconnecting processing elements (neurons)." These artificial neurons are connected together to form neural networks. An extremely simple example of such a network is shown in Figure 9. In this example, a number of inputs are each connected to each of a number of neurons in an intermediate layer. The neurons in the hidden layer are each connected to all the output neurons (one in this case). This is an example of a fully connected feed forward neural network. Feed forward networks of this type can be trained by back propagation. This is a procedure that trains the network by making small adjustments to the weights of each neuron in the direction that reduces the error at that neuron's output. The input layer used white blood cell feature 15 features. The hidden layer was designed by 5 nodes. Finally, the results of output layer were equal the number of white blood cells in different classes and determined from the probability of class membership. The whole process can be schematized for training process and testing process as showed in Figure 10 and 11 , respectively.

\section{Results and Discussion}

Fig. 12 shows that the discrimination between nuclear and nonnuclear pixels is selected after an automatic threshold value. As seen in Table 2, these leukocytes features is determined from the basis of the feature set. These parameters can be used as efficient features for inputs of classifiers. The test applied these leukocytes features are carried out and the results are shown in Table 3. The results presented here are based on trials conducted with normal cells. For training the classifiers, a library set of 233 patterns is used. The tested data consists of 134 samples and produced correct classification rate close to $88.10 \%$

\section{Conclusions}

This paper presented a methodology to achieve a fully automated detection and classification of leucocytes by microscope color images identifying the following classes: Basophil, Eosinophil, Lymphocyte, Monocyte and Neutrophil. Experiments show that the final classification module implemented by means of a parallel classifier composed by back propagation neural classifiers achieves an accurate solution with minor computational complexity than traditional nearest neighbor classifier. Results indicate that the morphological analysis of blood's white cells is achievable and it offers remarkable classification accuracy.

Further studies will be focused on other methods of determining the boundaries of cells. The method chosen here was, perhaps, a little naïve and worked poorly in a number of instances. This is the only step in the process where manual intervention was required. Hence, it is the only block to the automation of the entire process from data acquisition (which could now have been performed automatically if an automated microscope stage was affordable or at hand), through white cell segmentation, to classification. Counting the classified instances is seen as trivial. And, use of other features in the blood cell. Some of these features will require higher resolution images. In particular, the images acquired for this research were of too 
low a resolution to show the characteristic granularity that is a key element in the haematologist's differentiation of the granular leukocytes (neutrophils, eosinophils and basophils from lymphocytes and monocytes. What's more, Extension to other types of white blood cells. Blast cells, for instance, are characteristic of certain types of Leukemia and would indicate further tests if found in blood. Being able to automatically classify these and flag samples accordingly could be a real boon to haematologists. This would of course require leukemic blood with these unusual cells in evidence to be available and some image acquisition (and manual classification by haematologists for the training set). And, tests of statistical significance. Tests of the statistical significance of the differences in performance of the different classification algorithms could be conducted, given a number of trials with different data sets.

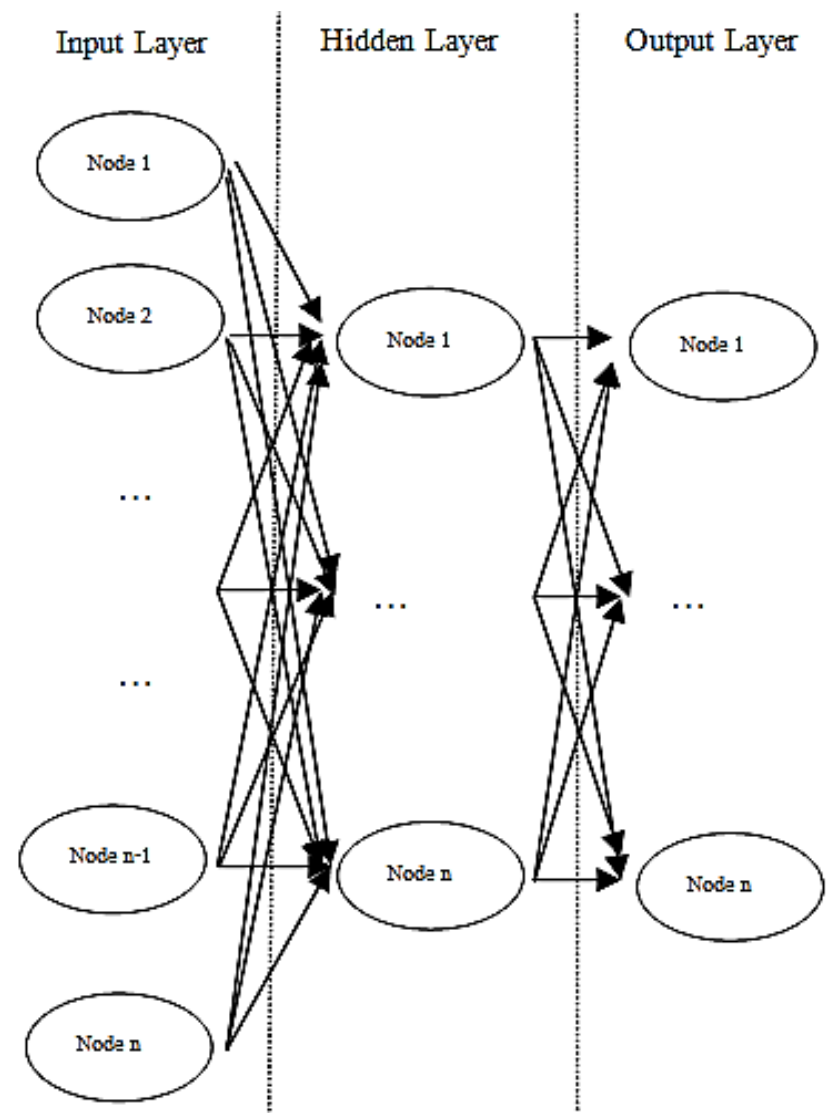

Fig.9. Architecture of a Three-layered Pattern Classification Neural Network.

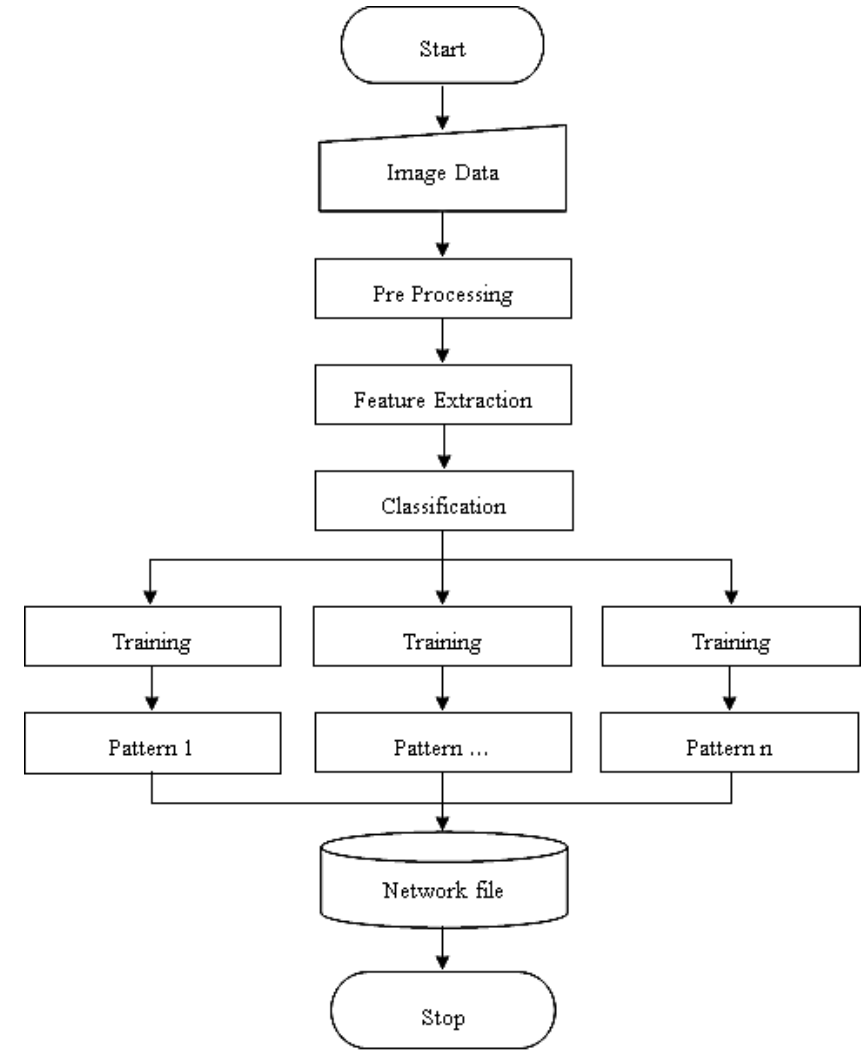

Fig.10. Training process.

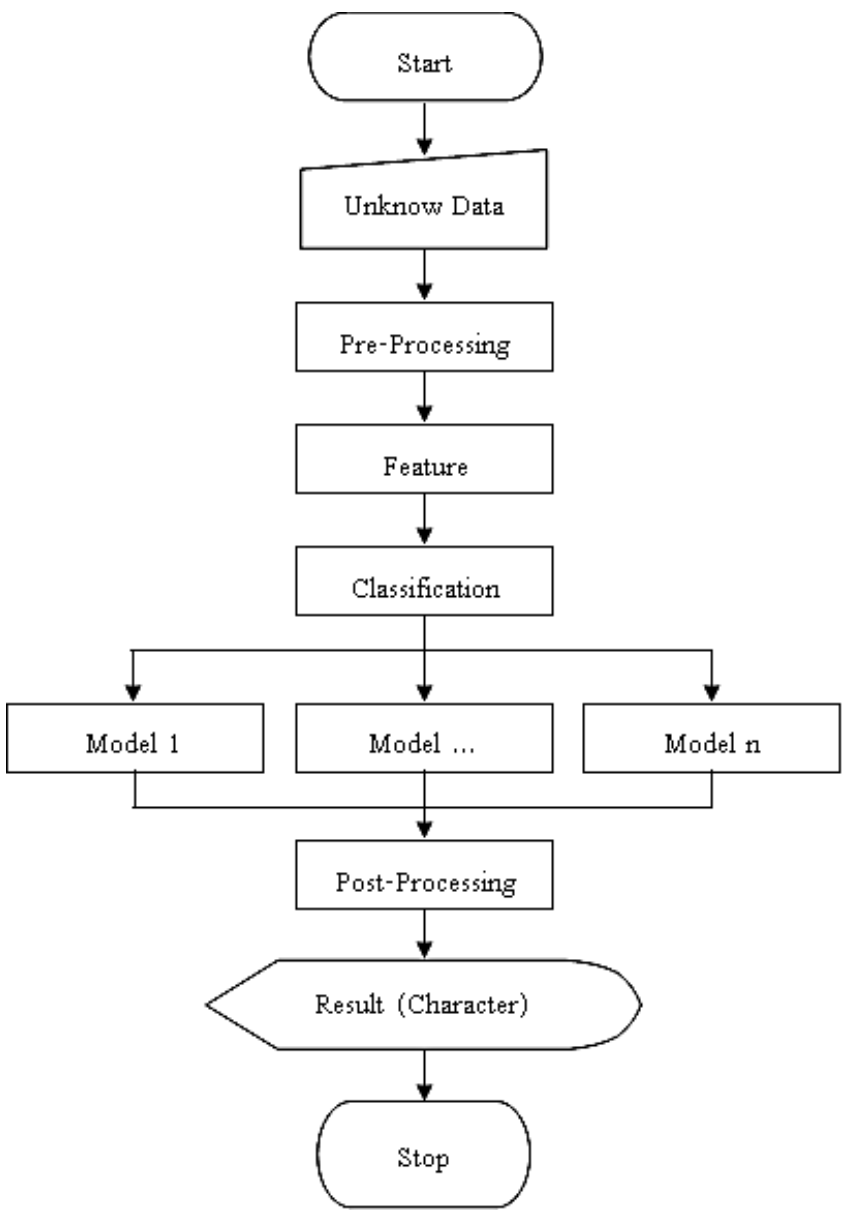

Fig.11. Testing process. 


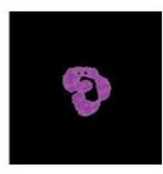

(a)

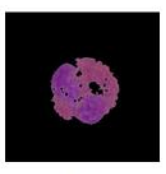

(b)

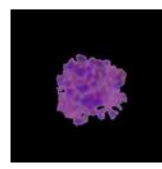

(c)

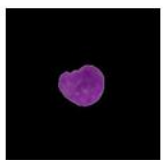

(d)

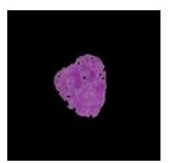

(e)

Fig. 12. The nucleus segmentation.

Table 2. The features of leukocytes.

\begin{tabular}{|c|c|c|c|c|c|c|}
\hline & \multicolumn{2}{|c|}{ Number of pixels in circle } & \multicolumn{2}{|c|}{$\begin{array}{c}\text { Number of circle edge } \\
\text { pixels }\end{array}$} & \multicolumn{2}{|c|}{ Number of nuclear pixels } \\
\hline & $\overline{\mathrm{x}}$ & S.D. & $\overline{\mathrm{X}}$ & S.D. & $\overline{\mathrm{x}}$ & S.D. \\
\hline Neu & 6808.31 & 1362.10 & 290.58 & 29.17 & 2426.45 & 533.36 \\
\hline Eosi & 12607.39 & 3747.76 & 393.17 & 60.45 & 4546.22 & 1388.94 \\
\hline Baso & 14357.71 & 4464.72 & 420.29 & 62.47 & 5700.71 & 925.84 \\
\hline Lym & 5084.51 & 942.12 & 251.25 & 23.19 & 2481.21 & 463.56 \\
\hline \multirow[t]{3}{*}{ Mono } & 10430.25 & 5068.42 & 355.58 & 65.98 & 3582.04 & 603.78 \\
\hline & \multicolumn{2}{|c|}{$\begin{array}{c}\text { Number of nuclear edge } \\
\text { pixels }\end{array}$} & \multicolumn{2}{|c|}{$\begin{array}{l}\text { Number of nuclear pixels/ } \\
\text { Number of pixels in circle }\end{array}$} & \multicolumn{2}{|c|}{$\begin{array}{c}\text { Number of nuclear edge } \\
\text { pixels/ Number of circle } \\
\text { edge pixels }\end{array}$} \\
\hline & $\overline{\mathrm{X}}$ & S.D. & $\overline{\mathrm{X}}$ & S.D. & $\overline{\mathrm{X}}$ & S.D. \\
\hline Neu & 655.02 & 276.75 & 0.36 & 0.04 & 2.24 & 0.86 \\
\hline Eosi & 1018.26 & 400.98 & 0.36 & 0.06 & 2.56 & 0.86 \\
\hline Baso & 982.14 & 535.10 & 0.41 & 0.06 & 2.24 & 0.90 \\
\hline Lym & 473.26 & 378.39 & 0.49 & 0.02 & 1.85 & 1.33 \\
\hline \multirow[t]{3}{*}{ Mono } & 798.72 & 276.92 & 0.370 .07 & 0.07 & 2.28 & 0.81 \\
\hline & \multicolumn{2}{|c|}{$\begin{array}{l}\text { Number of nuclear edge } \\
\text { pixels/ Number of nuclear } \\
\text { pixels }\end{array}$} & \multicolumn{2}{|c|}{$\begin{array}{c}\text { Number of circle edge } \\
\text { pixels/ Number of pixels in } \\
\text { circle }\end{array}$} & \multicolumn{2}{|c|}{$\begin{array}{c}\text { (Number of nuclear edge } \\
\text { pixels/ Number of nuclear } \\
\text { pixels)/ (Number of } \\
\text { circle edge pixels/ Number } \\
\text { of pixels in circle) }\end{array}$} \\
\hline & $\overline{\mathrm{X}}$ & S.D. & $\overline{\mathrm{X}}$ & S.D. & $\overline{\mathrm{X}}$ & S.D. \\
\hline Neu & 0.27 & 0.11 & 0.04 & 0.001 & 6.33 & 2.52 \\
\hline Eosi & 0.24 & 0.10 & 0.03 & 0.010 & 7.39 & 3.34 \\
\hline Baso & 0.17 & 0.07 & 0.03 & 0.001 & 5.85 & 3.39 \\
\hline Lym & 0.1 & 0.13 & 0.05 & 0.001 & 3.83 & 2.85 \\
\hline \multirow[t]{3}{*}{ Mono } & 0.2 & 0.10 & 0.04 & 0.001 & 6.52 & 2.79 \\
\hline & \multicolumn{2}{|c|}{ Red pixel value } & \multicolumn{2}{|c|}{ Green pixel value } & \multicolumn{2}{|c|}{ Blue pixel value } \\
\hline & $\overline{\mathrm{X}}$ & S.D. & $\overline{\mathrm{X}}$ & S.D. & $\overline{\mathrm{X}}$ & S.D. \\
\hline Neu & 12188791.40 & 82769.36 & 12359095.73 & 46540.65 & 12162288.74 & 81247.04 \\
\hline Eosi & 11800272.17 & 234635.82 & 12124605.65 & 134409.78 & 11834367.09 & 197198.01 \\
\hline Baso & 11721998.29 & 103397.88 & 12110284.43 & 72337.32 & 11657510.29 & 127915.85 \\
\hline Lym & 12181788.78 & 88758.59 & 12354375.56 & 53731.40 & 12138643.53 & 82304.77 \\
\hline Mono & 11913040.83 & 598702.58 & 12153595.91 & 596521.49 & 11874442.01 & 601881.29 \\
\hline
\end{tabular}

\begin{tabular}{|c|c|c|c|c|c|c|}
\hline & \multicolumn{2}{|c|}{$\begin{array}{c}\text { Green pixel value / Blue } \\
\text { pixel value }\end{array}$} & \multicolumn{2}{|c|}{$\begin{array}{l}\text { Green pixel value / } \\
\text { Red pixel value }\end{array}$} & \multicolumn{2}{|c|}{ Nuclear Texture } \\
\hline & $\overline{\mathrm{X}}$ & S.D. & $\overline{\mathrm{x}}$ & S.D. & $\bar{x}$ & S.D. \\
\hline Neu & 0.41 & 0.06 & 0.45 & 0.05 & 12.98185884 & 2.991436519 \\
\hline Eosi & 0.55 & 0.05 & 0.53 & 0.03 & 23.86688696 & 5.068758689 \\
\hline Baso & 0.46 & 0.04 & 0.49 & 0.04 & 22.76578014 & 2.466787328 \\
\hline Lym & 0.39 & 0.07 & 0.45 & 0.07 & 13.22287196 & 3.682092695 \\
\hline Mono & 0.46 & 0.06 & 0.50 & 0.06 & 17.98124036 & 3.637544634 \\
\hline
\end{tabular}

Table 3. The results of testing by leukocytes features set.

\begin{tabular}{|l|c|c|c|c|c|}
\hline \multirow{2}{*}{ Leukocytes } & \multirow{2}{*}{ Samples } & \multicolumn{2}{c|}{ Correct } & \multicolumn{2}{c|}{ Incorrect } \\
\cline { 2 - 6 } & & Numbers & $\%$ & Numbers & $\%$ \\
\hline Neutrophils & 39 & 35 & 89.74 & 4 & 10.26 \\
Eosinophils & 30 & 23 & 76.67 & 7 & 23.33 \\
Basophils & 5 & 4 & 80.00 & 1 & 20.00 \\
Lymphocytes & 30 & 30 & 100.00 & 0 & 0.00 \\
Monocytes & 30 & 26 & 90.00 & 4 & 10.00 \\
Total & 134 & 118 & 88.10 & 16 & 11.90 \\
\hline
\end{tabular}

\section{Acknowledgment}

This work is partially supported by Rangsit Research Institute at Rangsit University, Department of Telecommunications Engineering at King Mongkut's Institute of Technology Ladkrabang, and Department of Industry Physics and Medical Instrument at King Mongkut's University of Technology North Bangkok. The authors also gratefully acknowledge the helpful comments and suggestions of the reviewers, which have improved the presentation.

\section{References}

(1) F. Cillesen, W. Der Meer, Atlas of Blood Cell Differentiation, Elsevier Science B.V., Amsterdam The Netherlands. 1998.

(2) Keith Breden Taylor and Julian B. Schorr, Blood, Colliers Encyclopaedia. vol. 4 (1978).

(3) A. Hughes, A History of Cytology, New York: Aberlard-Schuman, 1959.

(4) G. A. Daland and T. H. Ham, A Color Atlas of Morphologic Hematology, Cambridge, Mass.: Harvard University Press. 1967.

(5) R. S. Ledley, High-speed automatic analysis of biomedical pictures, Science. October (1964) 216-223.

(6) P. G. Stein, L. E. Lipkin, and H. M. Shapiro, Spectra II: general-purpose microscope input for a computer, Science. vol. 166 (1969) 328- 333. 
(7) J. W. Butler, Automatic analysis of bone autoradiographs, in Pictorial Pattern Recognition, G. C. Cheng et. al., Eds. Washington, D.C.: Thompson Book Co., 1968, pp. 75-85.

(8) M. R. Evans and J. W. Sweeney, A computer technique for investigating and rationalization of scintillation scan reading, Methods Inform. Med. vol. 6 no. 1 (1967) 24-27.

(9) F. Winsberg et al., Detection of radiographic abnormalities in mammograms by means of optical scanning and computer analysis, Radiology. vol. 89 (1967) 211-215.

(10) G. L. Wied et al., Taxonomic intracellular analytic system (TICAS) for cell identification, Acta. Cytol. vol. 12(1968) 180-204.

(11) J. W. Butler, M. K. Butler, and A. Stroud, Automatic classifications of chromosomes-II, in 1964 Proc. Rochester Conf. Data Acquisition and Processing in Biology and Medicine. New York: Pergamon, 1965.

(12) J. Hilditch and D. Rutovitz, Chromosome recognition, Ann. N.Y. Acad. Sci. vol. 157 (1969) 339-364.

(13) Pramit Ghosh, Debotosh Bhattacharjee, Mita Nasipuri and Dipak Kumar Basu, Automatic White Blood Cell Measuring Aid for Medical Diagnosis. Process Automation, Control and Computing (PACC). 20-22 July (2011) 1-6.

(14) Sawsan F. Bikher, Ahmed M. Darwish, Hany A. Tolba, Samir I. Shaheen. Segmentation and Classification of White Blood Cells. IEEE International Conference on Acoustics, Speech, and Signal Processing. vol. 6 5-9 June (2000) 2259 - 2261.

(15) Neelam Sinha, A.G.Ramakrishnan. Automation of Differential Blood Count. TENCON 2003: Conference on Convergent Technologies for the Asia-Pacific Region. vol. 2 15-17 Oct. (2003) 547 - 551.

(16) I. Cseke, "A fast segmentation scheme for white blood cell images," in Proceedings of the $11^{\text {th }}$ IAPR International Conference on Pattern Recognition, Conference C: Image, Speech and Signal Analysis, pp. 530-533, 1992.

(17) J. W. Bacus, "An automated classification of the peripheral blood leukocytes by means of digital image processing," Ph.D. dissertation, Univ. Illinois, Chicago, 1970.

(18) [2] C. W. Barnett, "The unavoidable error in the differential count of the leukocytes of the blood," J. Clin. Invest., vol. 12, pp. 77-85, 1933.
(19) J. W. Butler, M. K. Butler, and A. Stroud, "Automatic classifications of chromosomes-II," in 1964 Proc. Rochester Conf. Data Acquisition and Processing in Biology and Medicine. New York: Pergamon, 1965.

(20) J. W. Butler, "Automatic analysis of bone autoradiographs," in Pictorial Pattern Recognition, G. C. Cheng et. al., Eds. Washington, D.C.: Thompson Book Co., pp. 75-85, 1968.

(21) T. D. Caspersson, G. Lomakka, G. Svenson, and R. Saftstrom, "A versatile ultramicrospectrograph for multiple-line and surface scanning high resolution measurements employing automated data analysis," Expt. Cell Res., vol. 3, pp. 40-51, 1955.

(22) H. J. Conn, Biological Stains, 7th ed. Baltimore, Md.: Williams and Wilkens, 1961.

(23) G. A. Daland and T. H. Ham, A Color Atlas of Morphologic Hematology. Cambridge, Mass.: Harvard University Press, 1967.

(24)M. R. Evans and J. W. Sweeney, "A computer technique for investigating and rationalization of scintillation scan reading, "Methods Inform. Med., vol. 6, no. 1, pp. 24-27, 1967.

(25) J. Hilditch and D. Rutovitz, "Chromosome recognition," Ann. N.Y. Acad. Sci., vol. 157, pp. 339-364, 1969.

(26) A. Hughes, A History of Cytology. New York: Aberlard-Schuman, 1959.

(27) M. Ingram, P. E. Norgren, and K. Preston, Jr., "Automatic differentiation of white blood cells," in Image Processing in Biological Sciences, D. M. Ramsey, Ed. Berkeley, Calif.: Univ Calif. Press, 1968.

(28) M. Ingram and K. Preston, Jr., "Automatic analysis of blood cells," Sci. Amer. vol. 223, pp. 72-82, 1970.

(29) L. W. Koster, "Color photography of biological stains," J. Biol. Photogr. Ass., vol. 35, no. 1, Feb., 1967.

(30) R. S. Ledley, "High-speed automatic analysis of biomedical pictures," Science, pp. 216-223, Oct. 1964.

(31) M. L. Mendelsohn, B. H. Mayall, J. M. S. Prewitt, R. C. Bostrom, and W. G. Holcomb, "Digital transformations and computer analysis of microscopic images," in Advances in Optical and Electron Microscopy, V. E. Coslett, Ed. New York: Academic Press, 1968, pp. $77-150$.

(32) N. J. Nilsson, Learning Machines. New York: McGraw-Hill, 1965.

(33) J. M. S. Prewitt and M. L. Mendelsohn, "A general approach to image analysis by parameter extraction," in Proc. Computers in Radiology, Chicago, 1966. 
(34)P. G. Stein, L. E. Lipkin, and H. M. Shapiro, "Spectra II: general-purpose microscope input for a computer," Science, vol. 166, pp. 328- 333, 1969.

(35) G. L. Wied et al., "Taxonomic intracellular analytic system (TICAS) for cell identification, Acta. Cytol., vol. 12, pp. 180-204, 1968.

(36)F. Winsberg et al., "Detection of radiographic abnormalities in mammograms by means of optical scanning and computer analysis," Radiology, vol. 89, pp. 211-215, 1967.

(37)I. T. Young, "Automated leukocyte recognition," Ph.D. dissertation, Massachusetts Inst. Technol., Cambridge, 1969. 